\title{
Die lichtabhängige Besiedlung von Hafenstützpfeilern durch sessile Tiere und Algen aus dem Korallenriff bei Eilat (Rotes Meer)*
}

\author{
H. SCHUHMACHER \\ Rubr-Universität Bochum, Lebrstubl für Spezielle Zoologie; \\ Bochum, Bundesrepublik Deutschland
}

\begin{abstract}
The light-dependent colonization of pillars by sessile animals and algae from the coral reef at Eilat (Red Sea). The influence of light on coral reef communities has been studied in the merchant harbour of Eilat (Red Sea), where other parameters of the local ecosystem remain relatively constant. The harbour pier is supported over a length of about $550 \mathrm{~m}$ by eight rows of concrete pillars. Water current is parallel to the pillar rows. Benthic. animals and algae settle on the pillars, between the sun-exposed first and the extremely shadowy eighth row, according to the light gradient (about $13-0.1 \%$ of the light intensity above the surface). The observations were carried out from January, 1970 to March, 1971. In the most common species (28 animals and five algae), four settlement zones can be distinguished: (a) "bright zone" (13-3\% of the light intensity measured above the water surface) with Tridacna maxima, T. squamosa and Padina pavonia and some stone corals as indicators; (b) "dim-light zone, bright" (3-1\%) with Siphonochalina siphonella and Dendronephthya species as indicators; (c) "dim-light zone, dark" (1 - 0.4\%) with Acabaria biserialis and Clathraria rubrinodis as indicators; (d) "dark zone" $(0.4-0.1 \%)$ with violet Lithothamnion and Lithopbyllum species as indicators. The distribution of animals in the horizontal light gradient (rows 1-8) corresponds to that in the vertical light gradient in coral reefs; this has been verified by SCUBA diving to $40 \mathrm{~m}$ depth near Eilat, Dahab, and Sharm el Sheikh on the Sinai coast, and Aqaba on the Jordanian coast of the Gulf. Light intensity is the dominant ecological factor which affects the distribution of the majority of species in the reef.
\end{abstract}

\section{EINLEITUNG}

Die oft sehr differenzierte Verteilung der Tierformen des Korallenriffs wurde erst in jüngster Zeit bekannt, weil die Bearbeitung dieses Problemkreises Tauchuntersuchungen im Biotop voraussetzt. Nach der Feststellung der Verschiedenheit im Verteilungsbild der riffbewohnenden Arten stellt sich die Frage, warum einzelne Arten gerade hier und nicht andernorts im Riff angesiedelt sind. Bei dem Versuch einer Klärung stößt man auf ein zunächst unüberschaubares Netzwerk von sich gegenseitig be-einflussenden ökologischen Faktoren sowie von inter- und intraspezifischen Beziehungen, welche in ihrer Gesamtheit den jeweiligen Ansiedlungspunkt bestimmen.

* Mir Unterstützung der Deutschen Forschungsgemeinschaft. 
Tatsächlich ist dieses Okosystem so komplex, daß es bisher höchstens in Ausnahmefällen gelungen ist, in cinem voll ausgebildeten, „blühenden“ Korallenriff den "Stellenwert" eines bestimmten ökologischen Parameters bei gleichzeitiger Einwirkung der vielzähligen anderen Faktoren zu bestimmen.

Unter "Stellenwert" wird hier die Bedeutung eines Umweltfaktors im abwägenden Vergleich mit den übrigen Faktoren für die Existenz eines Individuums oder eines Kollektivs verstanden. Der Stellenwert eines Parameters, bezogen auf eine bestimmte Art, wird als gering angesehen, wenn sie diesem gegenüber eine große Toleranzbreite hat. Indikatorarten für die verschiedenen Umwelteinflüsse lassen somit in ihrer Verteilung im Riff Rückschlüsse über die im Biotop wirksamen ökologischen Faktoren zu.

Wie jung noch gerade der in situ operierende $Z$ weig der Riffökologie ist, wird besonders deutlich, wenn man zu dem Problem der Indikatorarten den heutigen Kenntnisstand in der Süßwasserökologie vergleicht. So schuf z. B. KolkwrTz bereits im Jahre 1909 mit seinem Saprobiensystem die Grundlage für eine auf Indikatorarten basierende biologische Beurteilung und Zonierung von Fließgewässern. Auf die bis heute erfolgte Verfeinerung und Ausweitung dieser Methode soll hier nicht weiter eingegangen werden.

Dagegen reicht zum gegenwärtigen Zeitpunkt der Kenntnisstand über Aussagewert und -genauigkeit einer im Korallenriff lebenden Art in den allermeisten Fällen noch nicht aus, um diese gegebenenfalls als Indikatorart für bestimmte ökologische Parameter heranziehen zu können.

Für das Rote Meer liegen bereits Beschreibungen einzelner Riffzonen auf korallensoziologischer Basis durch Loya $(1971,1972)$ und physiographisch-aspektorientierter Art durch KLAUSEWTTZ (1967) und MERGNER (1971) vor oder sind in Vorbereitung (MERGNER \& SCHUHMACHER 1973). Spezielle analytische Untersuchungen zur Riffökologie fehlen allerdings noch - nicht zuletzt wegen der eingangs aufgezeigten methodischen Schwierigkeiten.

Einer der wichtigsten standortbestimmten Faktoren ist das Licht. Bei der im Riff zu beobachtenden Artenverteilung von oben nach unten spielt die unterschiedliche Helligkeit sicher eine primär ursächliche Rolle. So sind bekanntermaßen die riffbildenden Korallen selbst wegen ihres Gehaltes an symbiontischen Zooxanthellen auf relativ geringe Wassertiefen beschränkt (zusammenfassende Übersicht z. B. bei YoNGE 1963). Es bleibt nur die Frage, inwieweit Wasserbewegung, Sauerstoffgehalt, Trübstoffbelastung, Wasserdruck und andere Faktoren den jeweiligen Standort der Korallen und übrigen Benthosarten mitbestimmen.

Im Laborexperiment würde man bei einer derartigen Fragestellung eine Lichtorgel einsetzen, um unter konstanten übrigen Bedingungen allein die Wirkung unterschiedlicher Lichtintensität beobachten und messen zu können. Allerdings gibt es für ein derartiges Großexperiment im Korallenriff selbst noch keine speziellen Anlagen. In Ermangelung von solchen wurde geprüf, ob nicht für andere Zwecke erstellte Kunstbauten als Lichtorgel nutzbar seien.

\section{UNTERSUCHUNGSORT UND METHODIK}

In Eilat können die Stützpfeiler des Handelshafens als eine Lichtorgel angesehen werden; sie wurden daraufhin während des gesamten Jahres 1970 und im Frühjahr 
1971 auf ihren Bewuchs untersucht. Zur Sicherung der Ergebnisse wurde der Besiedlungsstand im April 1972 noch einmal kontrolliert.

Die Kaimauer erstreckt sich von NNO nach SSW über eine Länge von ca. $550 \mathrm{~m}$ (Abb. 1). Sie wird von acht Reihen Stützpfeiler getragen. Diese sind aus Beton und von achteckigem Querschnitt. Jede Seite ist ungefähr $20 \mathrm{~cm}$ breit, der Durchmesser beträgt etwa $50 \mathrm{~cm}$. Der Abstand der Pfeiler liegt bei etwa 1,30 m. Die erste (äußerste) Pfeilerreihe steht $12 \mathrm{~m}$ tief im Wasser, die achte (ufernächste) reicht entsprechend der Böschung nur noch ca. $1 \mathrm{~m}$ tief ins Wasser, bezogen auf den mittleren Wasserstand. Zwischen Wasserspiegel und Unterseite der Kaiplattform ist noch ein Zwischenraum von ca. 1,20 m. Der Abstand von der äußersten Pfeileraußenseite bis zur Hafenmauer beträgt ungefähr $12 \mathrm{~m}$. Der Pier mit den Stützpfeilern wurde in den Jahren 1964 bis 1965, von Süden nach Norden fortschreitend, erbaut. Die Pfeiler standen im ausgewählten Untersuchungsabschnitt am Südende des Hafens bei Beginn der einjährigen Beobachtungen sechs Jahre im Wasser. Bereits im November des Jahres 1963 wurden südlich und nördlich der Kaimauer je drei Probepfeiler erstellt, die jetzt Leuchtfeuer tragen.

Das südliche Pierende war nur selten und dann nur kurzfristig von Schiffen belegt; daher wurden die regelmäßigen Beobachtungen und Probenentnahmen hier durchgeführt. Die im folgenden dargelegten Befunde entstammen in erster Linie eingehenden Untersuchungen des Bewuchses auf der sechsten bis zwölften Pfeilerquerreihe vor dem Südende (s. Abb. 1 B). Ergänzend hierzu wurde die von Süden unbeschattete letzte sowie auch die vorletzte Querreihe an der Südkante des Piers bearbeitet. Als Kontrollpunkt im freien Wasser wurde die südliche Probepfeilergruppe herangezogen. Wiederholte Stichproben am Norderende und mittleren Stellen des Piers bestätigten den repräsentativen Charakter des engeren Untersuchungsabschnittes.

Der Boden unter dem Hafenpier fällt ungefähr unter einem Winkel von $45^{\circ} \mathrm{zum}$ offenen Wasser hin $\mathrm{ab}$ und ist im Bereich der Pfeiler mit großen Steinblöcken oder Steinschutt bedeckt. Vor der ersten Pfeilerreihe erstreckt sich in 12-13 m Tiefe eine nicht mehr so steil nach Osten weiter abfallende Feinsand-Schlamm-Fläche. Hiervon ist das Wasser bis zu $2 \mathrm{~m}$ ïber Grund sichtbar stärker mit Trübstoffen belastet als in oberen Wasserschichten, und von hier rührt auch der dünne Schlickbelag, der die unbesiedelten Flächen der Steinblöcke bedeckt.

Die Sichtweite zwischen den Pfeilern lag immer ungefähr bei $12-15 \mathrm{~m}$, vor dem Pfeilerwald über der schlammigen Bodenfläche war sie erheblich geringer (ca. $8 \mathrm{~m}$ ). Die Wassertemperaturen während des Untersuchungszeitraumes sind in Tabelle 1 angegeben.

Tabelle 1

Verlauf der mittleren Monatstemperaturen im Untersuchungsgebiet in 2 bis $5 \mathrm{~m}$ Tiefe

\begin{tabular}{|c|c|c|c|c|c|c|c|}
\hline $\begin{array}{l}\text { Jahr } \\
\text { Monat }\end{array}$ & $\begin{array}{c}1970 \\
I\end{array}$ & II & III & IV & V & VI & VII \\
\hline Temperatur $\left({ }^{\circ} \mathrm{C}\right)$ & 22,2 & 21,9 & 21,5 & 22,2 & 22,4 & 22,9 & 24,5 \\
\hline $\begin{array}{l}\text { Jahr } \\
\text { Monat }\end{array}$ & $\begin{array}{l}1970 \\
\text { VIII }\end{array}$ & IX & $\mathrm{X}$ & XI & $\begin{array}{l}1970 \\
X I 1\end{array}$ & $\begin{array}{c}1971 \\
\text { I }\end{array}$ & II \\
\hline Temperatur $\left({ }^{\circ} \mathrm{C}\right)$ & 26,2 & 25,3 & 24,1 & 23,8 & 21,7 & 21,4 & 20,7 \\
\hline
\end{tabular}




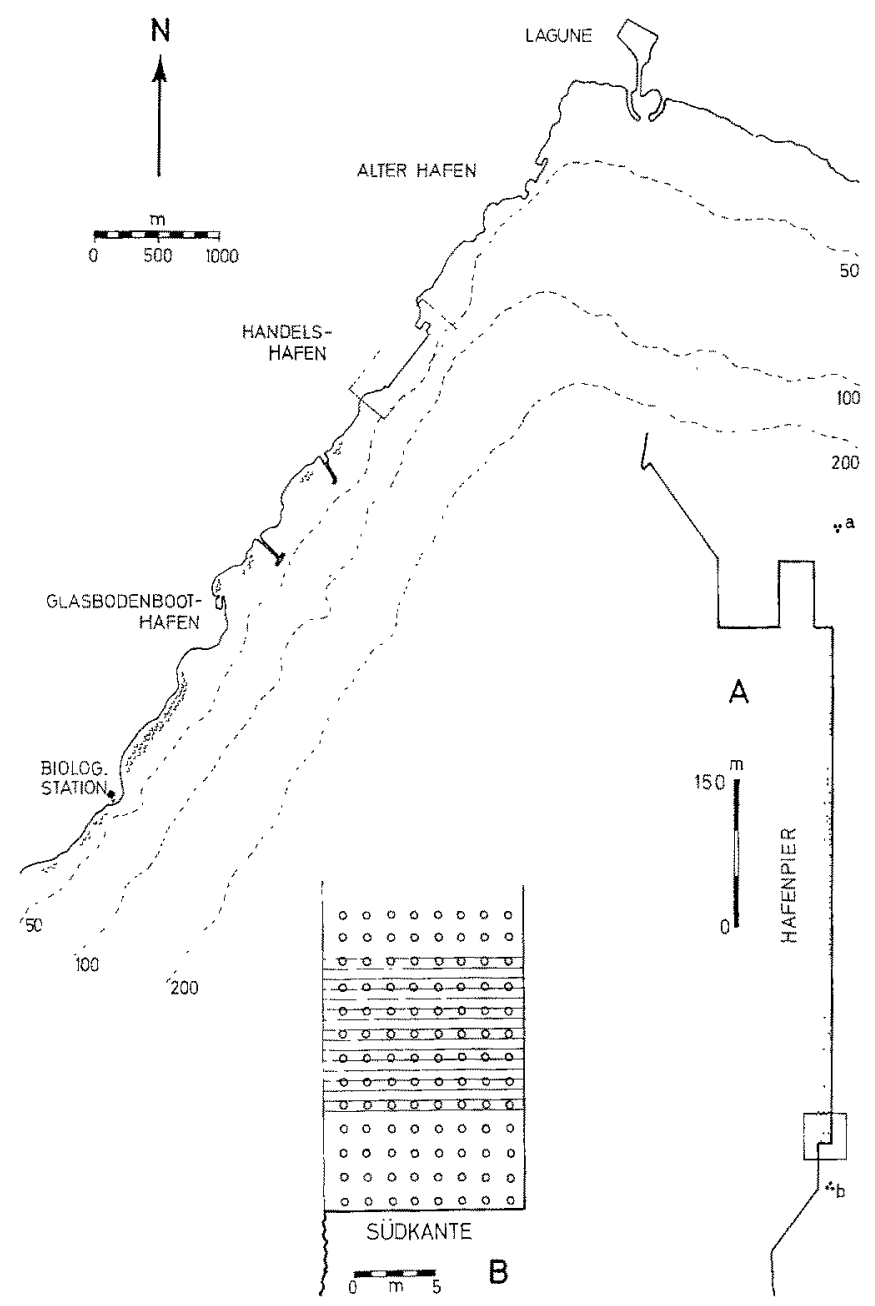

Abb. 1: Küstenverlauf bei Eilat mit Tiefenlizien, Angaben in $\mathrm{m}$; $A$ Handelshafen im Detail mit nördlichen (a) und südlichen (b) Probepfeilern; $B$ Ausschnitt aus dem Südende des Piers, engeres Untersuchungsgebiet schraffiert

Uber die Strömungsgesetzmäßigkeiten im Golf von Aqaba kann noch keine endgültige Aussage getroffen werden, ehe nicht Datenserien von einem Netz von Langzeitmeßstationen vorliegen. Einzelmessungen an der jordanischen wie Sinai-Küste des Golfes haben gezeigt, daß das Oberflächenwasser entsprechend dem fast ausschließlich aus nördlichen Richtungen wehenden Wind nach Süden getrieben wird. Dies gilt überwiegend auch für die bei Tauchabstiegen erreichten Tiefen von $30-40 \mathrm{~m}$. Vielfach wurde aber auch - an beiden Seiten des Golfes - in der Tiefe eine der Oberflächendrift entgegengesetzte Strömung längs der Küste nach Norden festgestellt, die bis einen halben $m$ unter der Oberfläche noch spürbar war und in ihrer Gesetzmäßigkeit (sicher u. a. gezeitenabhängig) noch nicht zu fixieren ist. 


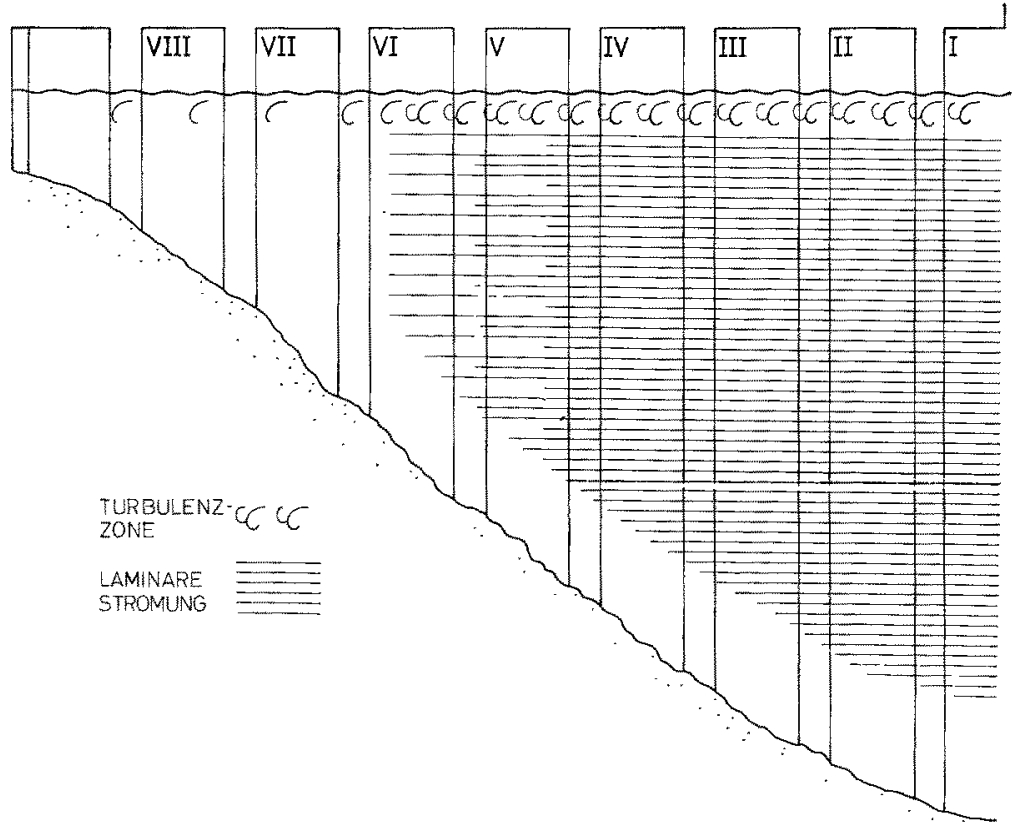

Abb. 2: Ost-West-Schnitt durch die Pfeilerreihen mit schematischer Darstellung der Wasserbewegung

In dem Pfeilerwald unter dem Hafenpier war daher - von ganz seltenen Fällen von Strömungsstille abgesehen - eine Strömung parallel zum Ufer aus nördlicher oder südlicher Richtung anzutreffen. Spizzengeschwindigkeiten von $10-12 \mathrm{~cm} / \mathrm{sec}$ wurden wiederholt gemessen, die meistens ermittelten Werte lagen bei 3-6 cm/sec. Diese Werte gelten für den in Abbildung 2 schraffierten Bereich. In Höhe der vierten Pfeilerreihe lag die Strömungsgeschwindigkeit noch bei $80 \%$, zwischen siebter und achter Reihe durchschnittlich nur noch bei 50\% der vor der Außenreihe gemessenen Werte. Die Strömungsgeschwindigkeit wurde mit Seidenpapier-Schnitzeln, die entlang einer Meßstrecke flottierten, ermittelt.

In einem Bereich bis zu ca. $70 \mathrm{~cm}$ unter dem Wasserspiegel wirken sich normalerweise die Schwappbewegungen und Turbulenzen aus, die entstehen, wenn sich die leicht schräg gegen die Uferlinie laufenden Wellen an den Pfeilern brechen.

Darunter (in Abb. 2 schraffiert gekennzeichnet) herrscht eine dem Genauigkeitsgrad der angewandten Meßmethoden entsprechend gleichmäßig laminare Strömung. (Die bei der langsamen Fließgeschwindigkeit unmittelbar am Substrat auftretenden schwachen Turbulenzen können hier vernachlässigt werden.) Dieser Bereich hat die von einer Lichtorgel zu fordernden Eigenschaften: Außer einer kontinuierlich abnehmenden BeIeuchtungsstärke sind die übrigen ökologischen Faktoren konstant. Auch hierbei gelten wieder die der angewandten Methodik entsprechenden Genauigkeitscinschränkungen. Nach den Erfahrungen vergleichender Tauchgänge an vielen Riffen des Golfes von Aqaba erscheinen jedoch die Aussagen der Hafenpfeiler-Lichtorgel bestätigt. 


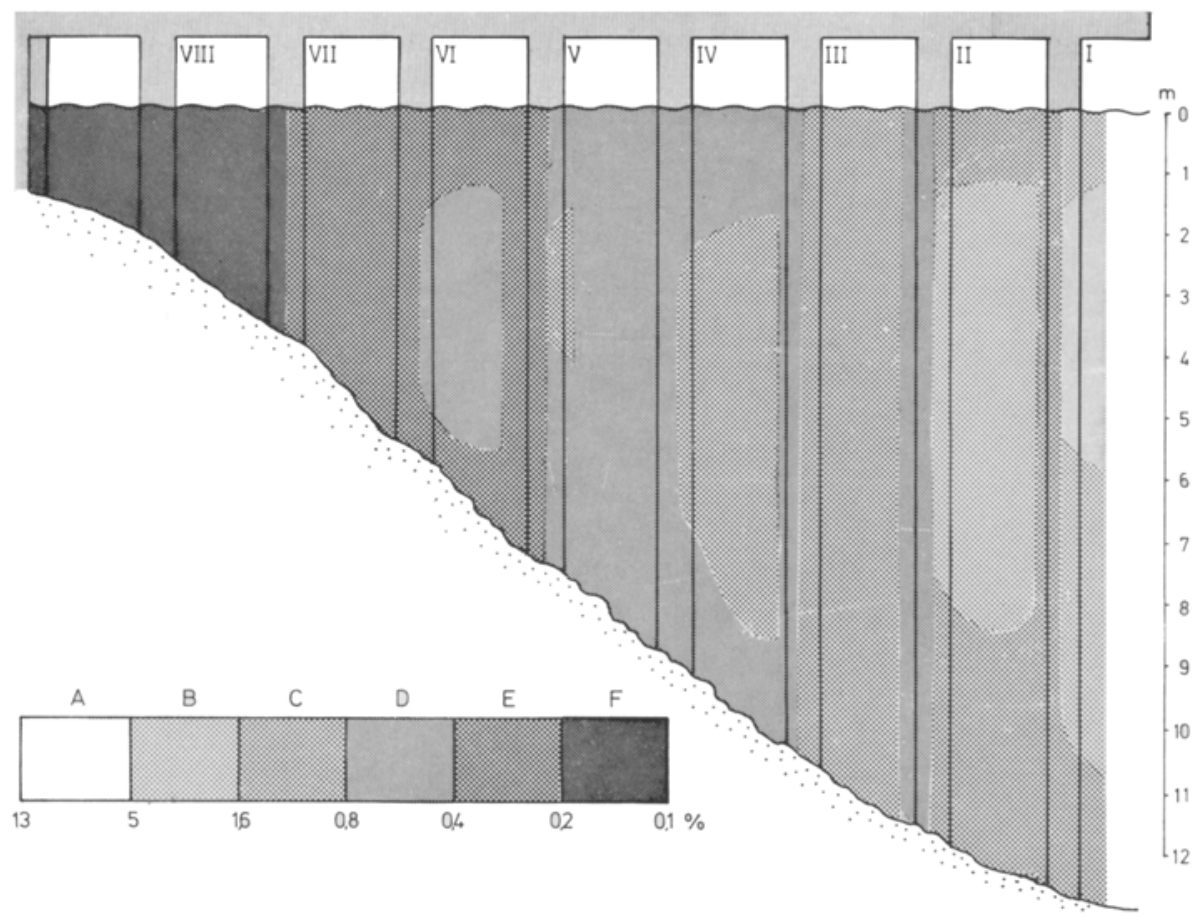

Abb. 3: Abfall der Beleuchtungsstärke im „Pfeilerwald“, dargestellt in sechs Stufen A-F

Die UW-Messungen der Beleuchtungsstärke wurden mit einem Lichtmeßgerät (Standard-Luxmeter, Fa. Lange, Berlin) und einem hiermit geeichten UW-Belichtungsmesser Gossen Sixtomat durchgeführt. Über die prinzipiellen Vorbehalte zu dieser vielfach angewandten Methode vergleiche JerLov (1970). Bei den Messungen konnte nicht zwischen einzelnen Spektralbereichen differenziert werden. Bei jedem Meßpunkt wurde (1) senkrecht nach oben, (2) waagerecht nach außen und (3) in Richtung des stärksten Lichteinfalls gemessen. Naturgemäß war letzterer an der Basis der Außenpfeiler nahezu senkrecht, im oberen Abschnitt der Außenpfeiler und bei den zwei nächstfolgenden Reihen schräg von oben und in der hinteren Hälfte des Pfeilerwaldes waagerecht von außen gerichtet. Nur die Außenseiten der ersten und stellenweise auch der zweiten Pfeilerreihe erhielten direktes Oberlicht, die anderen Pfeiler wurden nur von Reflexund Streulicht erreicht (vgl. auch RiEor 1966).

Die in Abbildung 3 angegebenen Prozentwerte sind auf die Beleuchtungsstärke bezogen, die über der freien Wasseroberfläche gemessen wurde. Es wurde demnach der unmittelbar über der Wasserfläche vor dem Pier gegen den Himmel gemessene Lichtwert gleich $100 \%$ gesetzt.

Der in Abbildung 3 dargestellte Gradient der Beleuchtungsstärke im Säulenwald entspricht einer Tageszeit zwischen 9 und 14 Uhr. Bei vormittäglichem Sonnenstand ist er zwischen den ersten Säulenreihen etwas steiler. Ein Unterschied zwischen Sommer und Winter macht sich hauptsächlich in einer erhöhten Zahl von bewölkten Tagen wäh- 
rend der Zeit von Ende November bis Ende Februar bemerkbar. Da die UW'-Lichtwerte zu den jeweiligen Werten über Wasser in Bezug gesetzt wurden, konnten diese Schwankungen der absoluten Beleuchtungsstärke nicht erfaßt werden.

Zählt man außer den festsitzenden Arten noch die auf den Pfeilern beweglichen benthischen Formen (Gastropoden, Polychaeten, Echinodermen etc.) und die Fische, die regelmäßig an oder zwischen den Pfeilern anzutreffen waren, hinzu, so würde eine Artenliste weit über hundert Namen umfassen, was die reichliche und vielfältige Besiedlung der Pfeiler kennzeichnet. Im folgenden wurden aus dieser Artenfülle nur diejenigen festsitzenden Formen ausgewählt, die in genügender Häufigkeit vorkommen, um Aussagen über eine mögliche Indikatoreignung machen zu können.

Gerade für vorliegende Fragestellung war der Untersuchungszeitraum, sechs bis acht Jahre nach Erstellung der Stïtzpfeiler, gut geeignet: Nur kurzfristig aufgetretene Erstbesiedler sind in der Zwischenzeit verdrängt worden; die jetzt vorgefundene Biocoenose erscheint soweit ausgewogen, daß sie als repräsentativ und nicht als kurzfristiges Zufallsprodukt gelten kann (zumindest waren von Anfang 1970 bis April 1972 keine größeren Verschiebungen im Artengefüge zu registrieren); andererseits haben proliferierende Arten mit der Tendenz, andere Formen zu überwuchern (wie z. B. Parerytbropodium fuloum oder verschiedene Xeniiden) noch nicht so entscheidend überhand genommen, daß letztlich die Biocoenose durch sie verarmt wäre.

\section{BEMERKUNGEN ZUR OKOLOGIE DER EINZELNEN ARTEN}

In Abbildung 4 ist die Verteilung von 28 Tier- und fünf Algenarten in dem Lichtgradienten unter dem Hafenpier angegeben. Eine ungefähre Vorstellung von der Verteilung dieser Arten auf den Stützpfeilern vermittelt Abbildung 5. Einzelheiten zum Vorkommen der verschiedenen Arten werden nachstehend berichtet.

Vergleichsbeobachtungen „,im Riff“ beziehen sich auf Tauchuntersuchungen, die vornehmlich im Naturreservat von Eilat zwischen Glasbodenboothafen und dem "Heinz Steinitz Marine Biology Laboratory" sowie unmittelbar südlich daron durchgeführt wurden. Weiterhin wurden jedoch auch Befunde von verschiedenen, gut ausgebildeten Riffen der Sinai-Küste bei Ras el Burka, Dahab und der Region von Sharm el Scheikh sowie von der jordanischen Golfküste herangezogen.

Siphonochalina siphonella bildet bis $30 \mathrm{~cm}$ lange graubeige Röhren, die dem Licht entgegenwadsen. Der Schwamm wurde allerdings nie auf stark belichteten Flächen gefunden - auch wenn, in Ausnahmefällen, das Endstück der Röhren zeitweilig besonnt sein kann, liegt doch der Anheftungspunkt des Stodkes stets in einem stark beschatteten Bereich. Die Art wurde hauptsächlich an der Rückseite der ersten und Vorderseite der $z$ weiten Pfeilerreihe in einer Tiefe von 0,5-7 m gefunden. Entsprechend belichtete Ansiedlungspunkte sind die Nischen zwischen den Steinblöcken von Hafenmolen, 1 bis $5 \mathrm{~m}$ tief, im Glasbodenboothafen und Jachthafen am Eingang der „Lagune" von Eilat. Typische Plätze im Riff sind für diesen Schwamm Kehlungen am Riffabsturz oder Nischen an der Basis von einzeln stehenden Korallenblöcken. Erst im Vorriff in $15 \mathrm{~m}$ Tiefe und mehr wächst er frei auf toten Korallen.

* Der Begriff „Vorriff“ wird unterschiedlich gebraucht. Hier wird unter Vorriff der von Korallen bedeckte Abhang unterhalb des Absturzes der äußersten Rifflkante angesehen. 
Callyspongia crassa (?) wurde auf den Probepfeilern im Schatten von Sarcopbyton glaucum und vereinzelt auf den Pfeilern von der ersten bis dritten Reihe gefunden. Im Riff ist die Art nicht aufgefallen.

Tubularia larynx fand sich in dichten Kolonien jeweils von Januar bis Mai/Juni an der Außenseite der ersten Pfeilerreihe bis zu $1 \mathrm{~m}$ unter der Wasserfläche, desgleichen einzelne Kolonien - in $50 \mathrm{~cm}$ Tiefe - an den südlichen Probepfeilern. Andere Fundorte waren die Unterseite eines $5 \mathrm{~cm}$ breiten Holztragegestells für Asbest-Besiedlungsplatten, welches außerhalb der „Lagune" an der Wasseroberfläche treibend verankert war. Dieses war Ende Januar 1970 binnen zwei Wochen in einer Länge von über $2 \mathrm{~m}$ unterseits völlig von Tubularia larynx bewachsen worden. Ein weiterer Fundort waren Steuerruder und Seitenwände des stationseigenen Forschungsschiffes im Glasbodenboothafen.

All diese Fundstellen waren nur mäßig beschattet, teilweise aber auch besonnt. Allen gemeinsam ist eine relativ heftige Wasserbewegung durch die anlaufenden Wellen und demgemäß eine mechanische Beanspruchung der hier angesiedelten Hydroiden.

Laomedea dichotoma wurde in lockeren Rasen ganzjährig auf der zweiten und dritten Pfeilerreihe gefunden; besonders häufig im obersten Bereidı bis zu $1 \mathrm{~m}$ unter dem Wasserspiegel - vergesellschaftet mit Acabaria pulchra.

Dynamena cornicina ist die häufigste Hydroidenart im Golf von Aqaba, wenn auch die Stöckchen nur einzeln auftreten. Mehr noch als an der äußersten Pfeilerreihe wuchsen Exemplare dieser Art an den hellexponierten südlichen Probepfeilern (zwischen 2 und $6 \mathrm{~m}$ Tiefe). Allerdings wurden auch einzelne Kolonien von der vierten und fünften Pfeilerreihe (in Nähe der Südkante der Pier) gesammelt. Im Gegensatz zu den vorgenannten Hydroiden scheint diese Art ruhiges oder gleichmäßig strömendes Wasser zu bevorzugen.

Halopteris glutinosa wurde jeweils nur im Frühjahr auf den beschatteten Pfeilern der zweiten bis vierten Reihe in verschiedenen Tiefen gefunden. Die am meisten lichtexponierte Kolonie saß auf einer Röhre von Siphonochalina sipbonella, die sich in $6 \mathrm{~m}$ Tiefe von der Rückseite des äußersten Pfeilers dem freien Wasser entgegenstreckte.

Millepora dichotoma - die augenfälligste Korallenform der Riffkante - fehlte bis auf zwei ca. $5 \mathrm{~cm}$ große Stücke an der Pfeileraußenreihe. Eine etwas größere Kolonie sitzt an einem der südlichen Probepfeiler. Zweifellos ist die Art sehr lichtbedürttig, da sie im Riff schon in 5-8 $\mathrm{m}$ Tiefe verschwindet.

Boloceroides momurrichi ist eine Aktinie, die sich durch Autotomie von Teilen der Mundscheibe mit Tentakel sehr rasch vegetativ vermehren kann. Die abgestoßenen Teile treiben zu neuen Siedlungsplätzen, wo sie zum kompletten Tier auswachsen. Daruber hinaus ist die Aktinie in der Lage, durch rhythmisches, gleichsinniges Eindrehen und Ausstrecken der Tentakel zu schwimmen. Es ist somit nicht nur Gewähr gegeben, ungünstige Standorte zu verlassen, sondern daruber hinaus auch die Möglichkeit, besser geeignete Plätze zuerst „auszuprobieren“.

Gerade wegen der leichten und raschen Vermehrung trift man die Art meist in größerer Anzahl an. Auf den ersten vier Pfeilerreihen bedeckten die Tiere of Flächen von jeweils bis $z \mathfrak{u} 30 \times 40 \mathrm{~cm}$. Am häufigsten im Bereich von 1-5 m Tiefe, fanden sie sich auch oft (in kleinen Ansammlungen oder einzeln) dicht unter der Ebbelinie oder bis hinunter zur Basis der Pfeiler. An den hintersten Pfeilerreihen saßen auch immer 


\section{Porifera}

1 Siphonochalina siphonella

2 Callyspongla crassa (2)

Cnidaria, Hydrozoa

3 Tubulata larynx

4 Laomedea dichotoma

5 Dynamene cornutina

6 Halopteris glutinosa

7 Mllepora dichotoma

Cnidaria, Hexacorallia

a Boloceroides momurrich

9 Triactis producta

10 Acropora, Pocillopora, Siylophora

Cnidaria, Octocarallia

11 Parerythropodium fuivum

12 Sarcophyton giaticum

13 Lithophyton arboreum

14 Dendronephitha, Stereonephthya

15 scleronephthya corymbosa

16 Nephthea albida

17 Xenia, Heferoxenia

18 Acabana pulchra

19 Acabaria biseriahs

20 Clathraria rubrimadis

Annelida, Polychaeta, Sedentaria

21 Salmacina spec.

Mollusca, Bivalvia

22. Tridacna maxima, squamosa

23 Chama, Spondylus

Arthropoda, Cirripedia

24 Tetrachta squamosa rufotincta

Tunicata, Ascidiacea

25 polycarpa mylihgera

26 Cnemidocarpa hemprichi

27 Pyuta momus

28 Botrylloldes magnicoecum nigrum Chlorophyta

29 Enteramorpha clathrata elongata

30 stypopodium zonale

Phaeophyta

31 Padina pavonia

32 Pocockiella vanegala

Rhodophyta

33 Lithothamnon, thophyllum

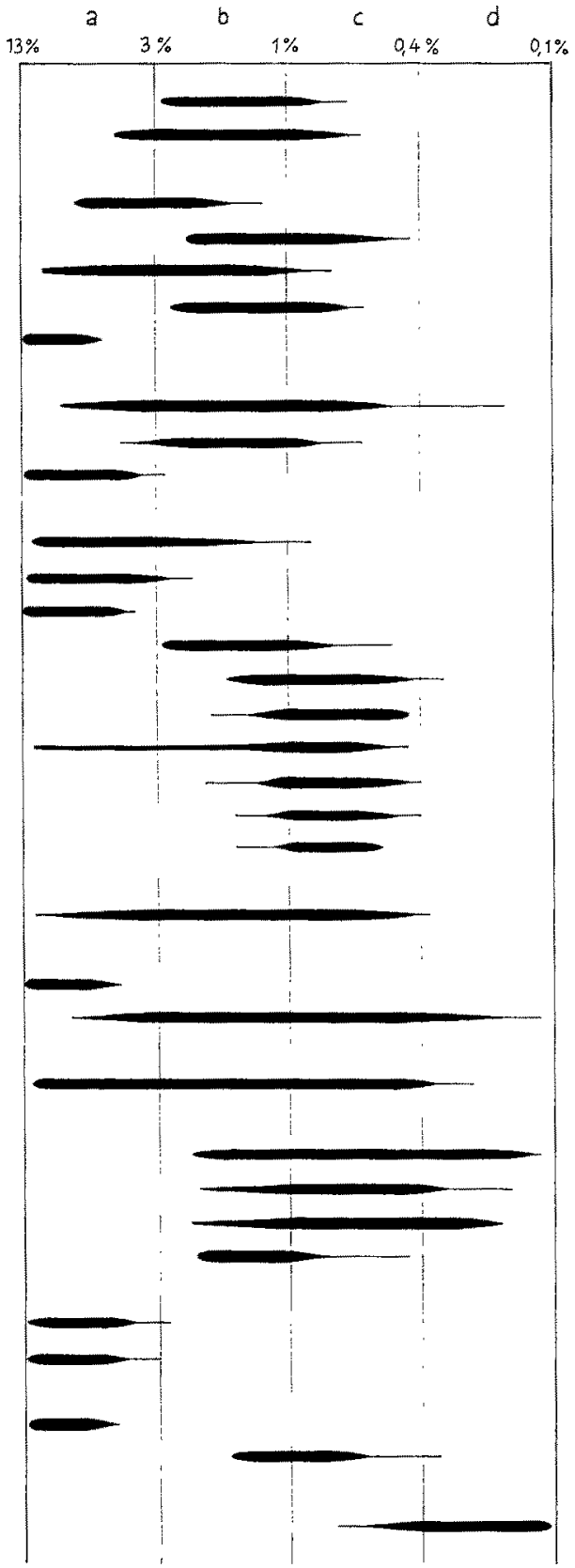

Abb. 4: Verteilung der häufigsten sessilen Tier- und Algenarten im Lichtgradienten der Stützpfeiler im Hafen von Eilat. Die Prozentwerte beziehen sich auf die unmittelbar über der freien

Wasserfläche gegen den Himmel gemessenen Helligkeit 
Einzeltiere, doch ist bei dieser halbsessilen Art schwer zu entscheiden, ob es sich hierbei nicht nur um temporäres Anhaften handelt. Beobachtungen im Riff, wo die Art gleichermaßen besonnte wie beschattete tote Korallenstücke besiedelt, bestätigen den Eindruck einer weiten Toleranz hinsichtlich des Lichtbedarfs.

Triactis producta ist im Riff sehr häufig, wenn auch unauffällig, da sie, meist zu vielen Exemplaren, in Nischen zwischen Korallenstöcken vorkommt. Dic Art hat einen ausgeprägten Tag-Nacht-Rhythmus: bei Dunkelheit wird ein bei Tag vollkommen eingezogener Tentakelkranz ausgefahren, mit dem wohl nächtliches Plankton gefangen wird. An den Pfeilern war Triactis producta nicht häufig; an der Außenreihe wurde die Art erst bemerkt, als sie sich nach dem Absterben der Steinkorallen unter deren Asten angesiedelt hatte. Vereinzelt war sie vorher schon auf Pfeilern der zweiten und vierten Reihe auf oder neben Spondylus-Schalen versteckt gefunden worden. Auch bei den Exemplaren der vierten Pfeilerreihe (wo es auch tagsüber sehr dämmrig war) waren die Tentakel tagsüber nicht zu sehen.

Stylophora pistillata, Acropora variabilis und Acropora scandens sowie Pacillopora favosa waren die auffälligsten Vertreter der Steinkorallen. Im Vergleich zum Riff spielten die Madreporaria in der die Pfeiler besiedelnden Lebensgemeinschaft keine große Rolle. Nur die Außenseite der ersten Pfeilerreihe empfängt genïgend Licht, daß sich Steinkorallen gegenüber den anderen Raumkonkurrenten behaupten können. Und auch hier fallen nur Zweigkorallen auf, die sich über das Substrat erheben; (zu Anfang) flächig wachsende "Gehirnkorallen" wie Cyphastrea microphtbalma, Favia pentagona, Platygyra lamellina werden von schneller wachsenden Konkurrenten in ihrer Ausdehnung behindert oder sind überwachsen worden.

Während im Korallenriff unmittelbar über dem Substrat durch dessen unregelmäßige Struktur und Form Wasserströmungen relativ stark abgebremst sind (mit Ausnahme des der Brandung unterworfenen Bereiches) sind die Hafenpfeiler, frei im bis zu $12 \mathrm{~m}$ tiefen Wasser stehend, voll dem hier herrschenden gleichmäßigen Wasserstrom ausgesetzt. Sie bieten insofern ein ideales Substrat für Filtrierer, die unter den für sie hier besseren Bedingungen die Steinkorallen von der sonst im Riff aspektbeherrschenden Position verdrängen können.

Daß sich für Steinkorallen die "Startbedingungen" inmitten dieser Konkurrenz mittlerweile noch verschlechtert haben, zeigt folgende Beobachtung:

Im August 1970 sind nahezu alle Zweigkorallen im südlichen Kaiabschnitt plötzlich abgestorben (die Ursache ist unbekannt, möglicherweise sind sie durch beim Beoder Entladen von Schiffen ins Wasser gekommene Stoffe geschädigt worden - wie z. B. Schwefelpulver und Phosphatstaub, dem Umschlag dieses Hafens entsprechend, regelmäßig ins Wasser gelangen). Bis April 1972 konnten nur recht vereinzelt inzwischen neu angesiedelte Korallenstöckchen festgestellt werden. Bei Parallelbeobachtungen an künstlichen Modellriffen sind im gleichen Zeitraum neu angesiedelte Stylophora pistillata und Acropora-Arten bis zu $3 \mathrm{~cm}$ Höhe herangewachsen.

Die toten Korallenzweige an den Außenpfeilern waren binnen zwei Wochen von kleinen fädigen Rot- und Braunalgen besiedelt, bis Ende des Jahres hatten vor allem die rasenbildende Aktinie Boloceroides momurrichi oder Xeniiden und Parerythropodium fulvum das neue Substrat besetzt.

Auf den allseits relativ stark belichteten nördlichen und südlichen Probepfeilern 
fanden die Steinkorallen bessere Lebensbedingungen - hier hatten sich insbesondere an den südlichen Probepfeilern binnen acht Jahren in 1-4 m Tiefe Stöcke von Stylophora pistillata (bis $18 \mathrm{~cm}$ Höhe bei $20 \mathrm{~cm}$ Durchmesser), Acropora scandens (bis $15 \mathrm{~cm}$ Höhe bei $12 \mathrm{~cm}$ Durchmesser) und Pocillopora favosa $(12 \mathrm{~cm}$ Höhe bei 10 bis $12 \mathrm{~cm}$ Durchmesser) angesiedelt. Kleinere Exemplare von Stylophora pistillata waren noch

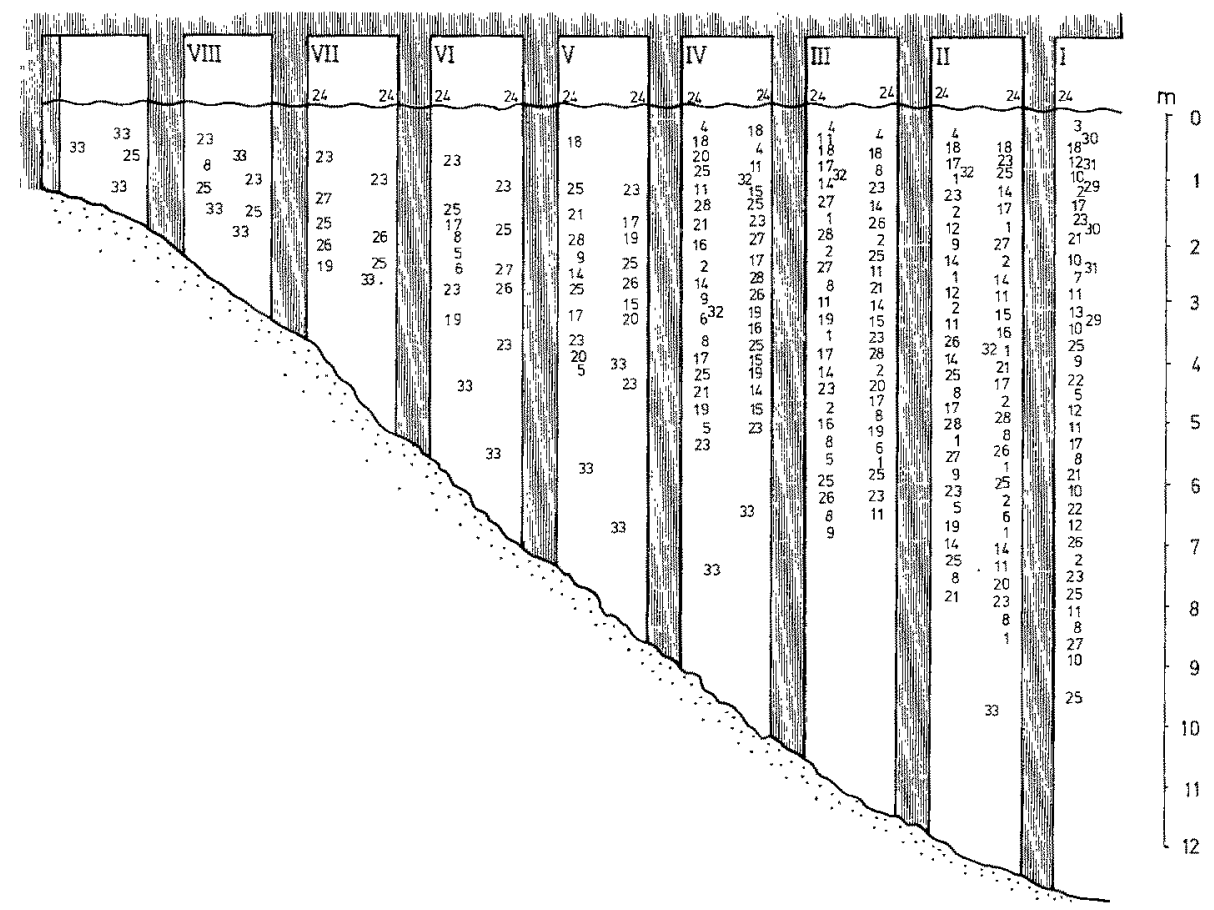

Abb. 5: Vereinfachte Darstellung der Besiedlung der Pfeiler durch folgende Arten: (1) Siphonachalina sipbonella; (2) Callyspongia crassa (?); (3) Tubularia larynx; (4) Laomedea dicbotoma; (5) Dynamena cornicina; (6) Halopteris glutinosa; (7) Millepora dichotoma; (8) Boloceroides mcmurrichi; (9) Triactis producta; (10) AcroporalPocillopora/Stylophora spec.; (11) Parerythropodium fulvum; (12) Sarcopbyton glaucum; (13) Lithopbyton arboreum; (14) Dendronephtbyal Stereonepbthya spec.; (15) Scleronephtbya corymbosa; (16) Nephthea albida; (17) XenialHeteroxenia spec,; (18) Acabaria pulcbra; (20) Clatbraria rubrinodis; (21) Salmacina spec.; (22) Tridacna maximalsquamosa; (23) CbamalSpondylus spec.; (24) Tetraclita squamosa rufolincta; (25) Polycarpa mytiligera; (26) Cnemidocarpa hemprichi; (27) Pyura momus; (28) Botrylloides magnicoecum/nigrum; (29) Enteromorpha clathrata elongata; (30) Stypopodium zonale; (31) Padina pavonia; (32) Pocockiella variegata; (33) Litbothamnion/Lithophyllum spec.

bis in $9 \mathrm{~m}$ Tiefe zu finden, tiefer waren Steinkorallen nur noch vereinzelt - und dann fast ausschließlich nur auf der Nordseite der Pfeiler - durch flächig wachsende „Gehirnkorallen" wie Anomastrea irregularis, Cyphastrea micropbthalma, Favia pentagona, Leptastrea transversa, Pavona maldivensis, Porites spec. $u$. a. vertreten. Überwiegend waren die drei Pfeiler in der unteren Hälfte durch Kalkalgen bedeckt oder ganz kahl (an der Basis in 12-13 m Tiefe). Es sei hier angemerkt, daß diese und andere Korallen- 
arten im Vorriff in wesentlich größeren Tiefen und bei geringerer Lichtintensität als an der Pfeilerbasis gefunden wurden.

Aspektbestimmend für die gesamte, auf den Pfeilern angesiedelte Biocoenose sind Oktokorallen, insbesondere Alcyonarien:

Parerythropodium fulvum kann sich als Kolonie rasch ausbreiten und dabei hauptsächlich tote Korallenstöcke überziehen, aber auch lebende Sedentarier zunehmend einengen und ersticken. Die Art hat keine sehr ausgesprochene Helligkeitspräferenz; sie war auf der ersten bis fünften Pfeilerreihe vertreten, allerdings sehr häufig nur auf den ersten beiden Reihen, wo Flächen bis zu einem halben qm ausschließlich nur von dieser Art bedeckt waren. Die Farbe von Sonnenlicht-exponierten Kolonien ist meistens ein dunkles Gelb, im Schatten wachsende Kolonien erscheinen mehr ockerfarben bis mittelbraun; im übrigen hängt die Färbung auch vom Kontraktionsgrad der Polypen ab. Im Riff wurde die Art vom Riffdach bis zu $40 \mathrm{~m}$ Tiefe gefunden, überwiegend jedoch am Riffabfall und im oberen Vorriff.

Sarcopbyton glaucum war im Hafen auf den drei äußeren Pfeilerreihen durch kleine bis mittelgroße (ca. $12 \mathrm{~cm}$ hohe) Exemplare nur vereinzelt, dagegen auf den Probepfeilern etwas häufiger vertreten. Im Riff zählt die Art bis zu ca. $20 \mathrm{~m}$ Tiefe zu den häufigen Formen; in Vertiefungen und auf flachen Abhängen des Riffdaches waren mancherorts mehrere qm einzig von dieser Species bestanden.

Litbopbyton arboreum hat eine ähnliche Tiefenverbreitung wie vorgenannte Art. Sie ist auf vielen Riffen des Golfs die häufigste Oktokoralle. Seltsamerwreise war sie nur durch zwei Exemplare auf der äußersten Pfeilerreihe (außen) vertreten; dagegen waren die drei nördlichen Probepfeiler von 1-4 m Tiefe gänzlich unter einem dichten Wald von Lithopbyton arboreum verborgen.

Dendronephtbya bemprichi und D. utinomii sowie Stereonepbtbya cundabiluensis zeigten sich in der Standortwahl streng lichtabhängig: während kein einziges Exemplar auf der Außenseite der ersten Pfeilerreihe Fuß gefaßt hatte, waren die Rückseite derselben Reihe und vor allem die zweite und dritte Reihe stellenweise dicht besetzt. Weiter hinten auf der vierten und füntten Pfeilerreihe waren die DendronepbtbyaArten nur noch vereinzelt vertreten. Während des Helligkeitsmaximums in den späten Vormittagsstunden hingen die vorderen Fächer schlaff herab. Dagegen waren die an den hintersten Pfeilern siedelnden Kolonien auch tagsüber teilweise in Fangstellung. Die Besiedlung der Pfeiler reichte von ungefähr $1 \mathrm{~m}$ unterhalb der Ebbelinie bis zu $7 \mathrm{~m}$ Tiefe.

Im Riff findet man die Dendronepbtbya-Arten in der Regel erst ab Tiefen unter $5 \mathrm{~m}$ und auch da nur unter Uberhängen und in beschatteten Kanälen. Ein beliebter Standort ist am Stamm der schirmförmig sich verbreiternden Acropora-scandens-Kolonien im oberen Vorriff. Tagsüber sind diese Dendronephtbya-Stöcke meist kontrahiert und entfalten sich erst am späten Nachmittag. In einer Tiefe von $20 \mathrm{~m}$ ist der Helligkeitswert soweit vermindert, daß sich hier die Kolonien auf strömungs-exponierten Korallenstöcken und Vorsprüngen entwickelten; diese Fächer ragen meistens auch tagsuber voll ausgestreckt in die Strömung. Nod tiefer $(30-40 \mathrm{~m})$ wurde Dendronephthya hemprichi und $D$. vitinomii regelrecht $z u$ großen Büschen gehäutt beobachtet (wie sie in solcher Dichte sonst nur auf den Hafenpfeilern auftraten).

Als passive Filtrierer sind diese Formen von einer gleichmäßigen Wasserströmung 
abhängig. Daher wird man sie nicht in strömungsstillen, wenn auch dunklen Höhlen, sondern vielmehr an „Hohlwegen“ oder unter Uberhängen am Riffabsturz finden, insbesondere wenn diese Formationen eine gewisse Düsenwirkung auf die entlangstreichende Strömung bewirken.

Scleronephthya corymbosa war auf den Hafenpfeilern wesentlich seltener als vorgenannte Nephtheiden vertreten. Die kleinen Scleronephtbya-Kolonien waren oft im Strömungsschatten - zwischen den größeren Dendronephthya-Stödken angesiedelt. In einer von 1970-1972 immer wieder, auch nachts besuchten Höhle (ca. $3 \mathrm{~m}$ unter der Wasseroberfläche, $3 \mathrm{~m}$ tief bei $2 \mathrm{~m}$ Offnungsdurchmesser) waren Decke und Wände dicht von dieser Art bestanden, während Dendronephtbya bemprichi $4 \mathrm{~m}$ entfernt Boden und Seitenwände eines kluftartigen Einschnittes besiedelt hatte. Auffallendster Unterschied zwischen beiden Standorten war, daß sich in der Kluft eine Strömung deutlich bemerkbar machte, während in der Höhle der Wasseraustausch wesentlich geringer war. Scleronephtbya corymbosa war tagsüber stets stark kontrahiert und dann von intensiv gelber Farbe. Abends und nachts "erblühten" die Kolonien regelmäßig zu drei- bis fünffacher Größe und glichen dann im Aussehen kleinen bis mittelgroßen Dendronepbthya-Stöcken von orange-rosa Färbung.

Nepbthea albida erinnert im Aussehen etwas an Lithopbyton arboreum. Daher wurden anfangs in 25-40 m Tiefe z. T. häufig auf Korallenstöcken wachsende Büsche dieser Art zunächst falsch angesprochen. Kleine Exemplare fanden sich in dunklen Nischen unter toten Korallenstöcken im Lagunenabschnitt des Riffdaches. Am Hafen $\mathrm{kam}$ die Art in 2 bis $3 \mathrm{~m}$ Tiefe auf der Rückseite der ersten und auf der dritten und vierten Pfeilerreihe vor.

Xeniidae spielen ebenfalls eine dominierende Rolle in der Pfeiler-Biocoenose. Es wurden mit Sicherheit die Arten Xenia macrospiculata, $X$. umbellata und Heteroxenia fuscescens identifiziert. Sicher sind noch weitere Species vertreten. Eine Verteilung der einzelnen Arten kann nicht angegeben werden, da sie - mit Ausnahme der Gattung Heteroxenia - im Leben kaum oder gar nicht zu unterscheiden sind. Xeniiden besiedelten von der ersten bis zur vierten Pfeilerreihe ähnlich große Flächen wie schon von Parerythropodium fuloum berichtet. Im Riff sind sie fast überall häufig. Nur auf dem Riffdach werden die brandungsexponierten Stellen etwas gemieden, dafür werden auch noch im tiefen Vorriff quadratmetergroße Rasenflächen gebildet. Inwieweit unterschiedliche Formen nur - eventuell standorttypische - Varietäten derselben Art oder verschiedene Arten darstellen, muß küntigen Freiwasseruntersuchungen vorbehalten bleiben.

Acabaria pulcbra war der häufigste Vertreter der Gorgonarien. Von der Rückseite der ersten bis zum fünften Pfeiler (mit Maximum auf der zweiten und dritten Reihe) war die Art bemerkenswerterweise nur im Turbulenzbereich der anlaufenden Wellen verbreitet. Die obersten $60 \mathrm{~cm}$ dieser Pfeiler waren überwiegend von Acabaria pulchra bestanden, darunter wurde die Anzahl der Stöckchen erheblich kleiner, bis unterhalb von 2,5 m kein Exemplar mehr zu finden war. Die Art ist vergesellschaftet mit der seltenen Acabaria erytbraea. Im Riff ist Acabaria pulchra häufig in den dunklen Kanälen und Gängen, die von der Riffkante in das Riffdach zurücklaufen. Außerdem findet man sie - ebenfalls auf dem Riffdach - unter abgestorbenen Korallenstücken oder Tridacnaschalen. Die Standorte haben alle gemeinsam, daß sie relativ dunkel und 
gleichzeitig durch anlaufende und sich brechende Wellen optimal mit Sauerstoff versorgt sind.

Acabaria biserialis hat zwar ungefähr den gleichen Lichtbedarf wie vorherige Art, aber sonst etwas andere ökologische Ansprüche: Von der Rückseite der zweiten bis fünften Pfeilerreihe waren die Kolonien von 2 bis $6 \mathrm{~m}$ ausschließlich senkrecht $z u$ der vorherrschenden laminaren Nord-Süd-Strömung ausgerichtet, um mit den in einer Ebene angeordneten Zweigen einen möglichst großen Filtriereffekt zu erzielen. Die Art trat in einer gelben oder orangeroten Varietät auf. Im Riff wurde sie in Tiefen von ca. $7 \mathrm{~m}$ an abwärts an gleichmäßig beströmten Standorten gefunden.

Clatbraria rubrinodis trägt ebenfalls in jungem Zustand die Zweige in einer Ebene quer zur Strömung ausgerichtet. Altere Kolonien wachsen aber dann dreidimensional weiter. Die Verbreitung auf den Pfeilern entspricht ungefähr der von Acabaria biserialis. Im Riff wurden in $20 \mathrm{~m}$ Tiefe bis zu. $30 \mathrm{~cm}$ hohe Kolonien gefunden.

Sedentäre Polychaeten, die vorläufig der Gattung Salmacina zugerechnet werden, waren häufig in mittleren Tiefen der ersten bis zur fünften Pfeilerreihe. Die ungefähr einen halben $\mathrm{mm}$ dicken Röhren sind, auch wenn sie zu mehreren zusammen gekittet sind, sehr zart und fragil und daher mechanischen Beanspruchungen durch Wellenschlag nicht gewachsen. Im Riff finden sich Salmacina-Kolonien dort, wo nur schwache Wasserbewegung gegeben ist, wie z. B. an der Basis von großen Korallenblöcken. Zweifelsohne ist bei diesen Polychaeten das Licht kein primär die Verbreitung bestimmender Faktor.

Das gilt dafür in besonders strengem Maße für die Muschelgattung Tridacna. Zwei Arten: Tridacna maxima und T. squamosa kommen im Golf von Aqaba vor. Beide sind äußerst lichtbedürftig und daher sehr häufig nur in seichtem Wasser auf dem Riffdach. Schon wenig tiefer als $5 \mathrm{~m}$ wurden sie nur noch in Ausnahmefällen gefunden. Die bestimmende Rolle der Helligkeit bei der Standortwahl zeigte sich auch bei der Besiedlung der Hafenpfeiler. Nur die hellsten Abschnitte der Außenseite (zwischen 1 und $5 \mathrm{~m}$ Tiefe) waren von diesen Muscheln besetzt. Zu Anfang der Untersuchungen wurden viele zwischen 12 und $15 \mathrm{~cm}$ lange Exemplare gemessen; diese groBen Stücke wurden aber durch Muschelsammler nach und nach entfernt. Vier von verschiedenen Pfeilern abgerissene Muscheln lebten am Fuß derselben in 12 bis $13 \mathrm{~m}$ Tiefe noch bis $z u$ zweieinhalb Monaten, danadh fanden sich nur noch die leeren Schalen, bzw. sie waren gänzlich verschwunden.

Die übrigen häufigen Muschelarten sollen summarisch besprochen werden, da keine ökologische Differenzierung zu bemerken war. Mehrere Arten der Gattung Chama sowie Spondylus aculeatus und S. gaederopus gehören zu den Erstbesiedlern der Pfeiler. Stellenweise erscheint die Oberfläche der Pfeiler wie genarbt von den alten festgewachsenen Schalenhälften. Diese organogene Kalkkruste toter oder noch lebender Muscheln ist bevorzugtes Substrat für Sekundärsiedler, insbesondere Oktokorallen. Wie weiter oben schon dargelegt wurde, ist der Pfeilerwald gerade für so spezialisierte Filtrierer wie die genannten Muscheln ein idealer Biotop. Daher sind die Pfeiler auch von der ersten bis zur sechsten Reihe dicht besiedelt; wohl wegen der zur Ufermauer hin geringer werdenden Strömung habetr sich dagegen an den letzten beiden Säulenreihen nur noch vereinzelt Exemplare von Spondylus gaederopus festgesetzt. Im Riff wurden die genannten Arten nicht so oft gefunden. Ein Grund hierfür liegt sicher in 
der guten Tarnung der Tiere durch Aufwuchs. Zweifellos haben aber auch die besonders günstigen Strömungsverhältnisse zu der ungewöhnlichen Siedlungsdichte auf den Hafenpfeilern geführt. Hinsichtlich Helligkeit stellen die Tiere keine spezifischen Anspriiche.

Tetraclita squamosa rufotincta wird in diesem Zusammenhang mit aufgeführt, weil die Art auf den Pfeilern sehr häufig ist, wenn sie auch nicht zur KorallenriftBiocoenose im strengen Sinne zu rechnen ist. Diese Seepocken besiedeln im Litoral den oberen Gezeitensaum und sind hier extremer Sonnereinstrahlung ausgesetzt. Wie ihr gleichmäßig häufiges Vorkommen auf den Pfeilerreihen I bis IV (dahinter sinkt die Besiedlungsdichte ab) jedoch beweist, spielt das Licht keine Rolle beim Festsetzen der Larven. Vielmehr muß das Substrat bei Flut starker Wellenbewegung ausgesetzt sein. Wo das nicht der Fall ist (hintere Pfeilerreihen oder flach auslaufende Strände), fehlt diese Art.

Ascidien waren als Filtrierer natürlich auch in mehreren Arten vertreten. Die häufigsten sind: Polycarpa mytiligera, Cnemidocarpa bemprichi, Pyura momus, Botrylloides magnicoecum und Botrylloides nigrum.

Die ersten drei Arten sollen wieder gemeinsam besprochen werden, da sie - alle solitäre Formen - keine bemerkenswerten Unterschiede in ihrer Verteilung aufweisen. Ausgesprochen helle Bereiche auf den beiden vorderen Pfeilerreihen wurden weitgehend gemieden. Da die Tiere in erster Linie auf den Gehalt an suspendierten Nahrungspartikeln im umgebenden Wasser angewiesen sind, fand man sie ziemlich gleichmäßig von den Basisabschnitten der vorderen Pfeiler bis zu den letzten Reihen, wo sie besonders auffielen, da diese Bereiche kaum von anderen Tieren besiedelt sind.

Botrylloides magnicoecum und B. nigrum sind Synascidien, die ähnlich wie manche schon genannte Coelenteraten flächig das Substrat überziehen. Sie wurden zunächst nur auf der zweiten bis fünften Säulenreihe gefunden (in 1 bis $6 \mathrm{~m}$ Tiefe). Erst nachdem die Korallen auf der Pfeileraußenseite abgestorben waren, besiedelten sie auch die Nischen zwischen den einzelnen toten Korallenästen. Auch diese beiden Arten scheinen direktes Sonnenlicht zu meiden.

Alle genannten Ascidien wurden im Riff an beschatteten Stellen des Riffdaches oder hauptsächlich im Vorriff gefunden. Typische Standorte waren Stamm und Unterseite tisch-oder schirmförmiger Acropora-Kolonien.

Algen sind in der Regel bei der Standortwahl mehr vom Licht abhängig als Tiere (vgl. Hellebust 1970). Andererseits ist die jahreszeitliche Fluktuation der Bestände wesentlich größer, was die Brauchbarkeit als Indikator (sei es für Lichtintensität oder andere Parameter) wieder einschränkt.

Die langfädige Enteromorpba clatbrata elongata hatte im Zeitraum Februar bis Anfang Mai die Korallenstöcke an den Probepfeilern sowie an der Pfeileraußenreihe stellenweise völlig in einen dichten grünen Schleier eingehüllt.

Stypopodium zonale war ebenso nur zu derselben Jahreszeit in den oberen, 2 bis $3 \mathrm{~m}$ tiefen Abschnitten der Probepfeiler sowie der Außenseite der ersten Pfeilerreihe vertreten.

Padina pavonia bildet im Frühjahr in sehr seichtem, stark durchlichtetem Wasser (auf dem Riffdach) oft viele Quadratmeter große Rasen. Am Hafen waren die geeigneten Lichtbedingungen für diese Algen nur an den hellsten Stellen der südlichen 
Probepfeiler und der Außenreihe der Stützpfeiler gegeben, wo vereinzelt die abgestorbenen Korallen besiedelt wurden.

Pocockiella variegata bevorzugt dagegen recht beschattete Plätze: Rückseite der ersten und zweite bis fünfte Pfeilerreihe, von der Wasseroberfläche bis zu ungefähr $6 \mathrm{~m}$ Tiefe. Man findet diese Rotalge vielfach unter Korallenblöcken oder TridacnaSchalen auf dem Riffdach oder unter Uberhängen am Riffabsturz.

Rotviolette Lager von Lithopbyllum- und Litbotbamnion-Arten waren auf den letzten drei Pfeilerreihen vorherrschend. Auch stark abgedunkelte Höllen im Rif: waren stets von gleichfarbigen Kalkalgen ausgekleidet; ausschließlich immer dann, wenn in den Höhlen keine Strömung vorhanden war. Die Annahme liegt nahe, daß es sich hierbei um die gleichen Arten wie auf den hinteren Pfeilern handelt. Angesichts der taxonomischen Schwierigkeiten bei dieser artenreichen Gruppe wurde kein Versuch einer näheren Bestimmung unternommen.

Theoretisch ist sicher manchen Arten aus beiden Gattungen ein hoher Indikatorwert hinsichtlich Lichtintensität beizumessen, denn neben dem Vorkommen in sehr dunklen Höhlen ist bekanntermaßen der Anteil von kalkproduzierenden Litbothamnion- und Lithopbyllum-Arten auf dem sonnenexponierten Riffdach beträchtlich.

\section{DISKUSSION}

Das aufgezeichnete Bild der lichtabhängigen Besiedlung der Hafenpfeiler soll nun unter der eingangs aufgeworfenen Fragestellung nach dem Indikatorwert einer Art betrachtet werden. Hierzu ist zunächst einmal die Toleranzbreite der zur Diskussion stehenden Art hinsichtlich einer bestimmten Beleuchtungsstärke zu prüfen. Weiterhin ist der Aussagewert, der aus der Verteilung der einzelner Arten in der HafenpfeilerLichtorgel geschlossen wurde, im natürlichen Rift zu verifizieren.

Nach der Zusammenstellung in Abbildung 4 sowie den zu den einzelnen Arten gemachten Anmerkungen wird zunächst deutlich, daß verschiedene Arten ausgesprochen unspezifisch hinsichtlich der Beleuchtungsstärke sind. Hierzu gehören Dynamena comicina, Boloceroides momurrichi, Xeniiden, Salmacina spec., Chama spec., Spondylus spec., Tetraclita squamosa, Polycarpa mytiligera und Pyura momus.

Die bei der Auswahl des Standortes vom Helligkeitsgrad abhängigen Arten lassen sich zunächst in zwei Gruppen einteilen: einmal die Formen, die mehr oder weniger intensive, direkte Sonnenbestrahlung benötigen. Hierher gehören alle Arten, die auf symbiontische Zooxanthellen angewiesen sind. Die andere Gruppe meidet weitgehend direktes Oberlicht und besiedelt beschattete Plätze. Je nach Intensität des einfallenden Streu- und Reflexlichtes wurden hier verschiedene Vergesellschaftungen vorgefunden. Entsprechend läßt sich eine weitere (dreifache) Unterteilung dieser Gruppe vornehmen. Insgesamt sind also in der Hafenpfeiler-Lichtorgel auf Grund unterschiedlicher helligkeitsabhängiger Besiedlung vier Zonen zu unterscheiden:

(a) Hellzone (direktes Oberlicht 13-3\% der über der Wasseroberfläche gemessenen Beleudhtungsstärke) mit den Leitformen Tridacna maxima und T. squamosa, Padina pavonia und verschiedenen Steinkorallen. Dieser Bereich wird regelmäßig von direktem Sonnenlicht getroffen. $\mathrm{Er}$ ist am genauesten gegenüber den anderen Licht- 
zonen abzugrenzen. Sehr spezifische Repräsentanten hierfür sind Tridacna maxima und Tridacna squamosa sowie höchstwahrscheinlich Millepora dichotoma (die Art wurde deshalb nicht unter den Leitformen genannt, weil sie auf den Hafenpfeilern zu wenig repräsentiert war; doch sprechen die Befunde im Riff für einen starken Lichtbedarf).

Steinkorallen gehören ebenfalls in diese Zone, doch haben sie eine größere Toleranzbreite; manche Arten sind im Vorriff noch in $30 \mathrm{~m}$ Tiefe $\mathrm{zu}$ finden. Zooxanthellen und deren Lichtbedarf sind bei all den genannten Gruppen der eigentliche Grund für ihre Beschränkung auf die hellsten Standorte. Viele Algen, darunter Enteromorpha clathrata elongata, Stypopodium zonale und vor allem die sehr häufige Padina pavonia, sind ebenfalls in ihrem Vorkommen auf diese Zone beschränkt.

Es muß hier darauf hingewiesen werden, daß der hellste Bereich des Riffes, das Riffdach, in seiner Lichtintensität nur unzulänglich durch die Hafenpfeiler-Lichtorgel wiedergegeben wird. MERgner (1971) gibt für das Riffdach bei Eilat 10\% und mehr der Überwasser-Helligkeit an, noch unveröffentlichte Messungen bei Aqaba ergaben 20-60\%. Während die Riffplattform über den ganzen Tag hinweg einer nahezu senkrechten Sonneneinstrahlung ausgesetzt ist, wird die Pfeileraußenreihe nur vormittags unter einem zunehmend kleineren Winkel von direktem Sonnenlicht getroffen. Das ist sicher mit eine Ursache, daß Millepora dichotoma an den Hafenpfeilern so wenig zu finden war.

Größere Helligkeit (bis fast $20 \%$ der Lichtmenge über Wasser) wurde an den oberflächennahen Abschnitten der freistehenden, nördlichen und südlichen Probepfeiler gemessen. Neben vereinzelten Millepora-Kolonien dominierten an den südlichen Probepfeilern in diesem Abschnitt Stylophora pistillata, Acropora scandens und andere Steinkorallen. Da die Probepfeiler aber ca. 50 bzw. $100 \mathrm{~m}$ von den Kaistützpfeilern entfernt stehen, wurden sie nicht unmittelbar in das Lichtorgel-System einbezogen. Auf der ersten Pfeilerreihe waren die Steinkorallen lichtmäßig zwar nicht im Pessimum, aber gegenüber der Konkurrenz von weitgehend lichtindifferenten Filtrierern, die optimale Strömungsbedingungen vorfanden, benachteiligt. Bei Lichtverhältnissen, wie sie auf der Außenseite der zweiten Pfeilerreihe gegeben sind, kommt es am Fuß des Riffabsturzes oder im oberen Vorriff noch zu einer reichhaltigen Hexakorallenfauna. Da aber dort die auf den Hafenpfeilern ausnahmsweise so erfolgreichen Raumkonkurrenten normalerweise auch keine optimalen Bedingungen finden, bleibt der Madreporarien-Aspekt vorherrschend.

Die in Abbildung 4 wiedergegebene Beschränkung von Lithophyton arboreum auf den Helligkeitsbereich (a) (gestützt auf das Vorkommen an den nördlichen Probepfeilern) entspricht nicht ganz der Realität im Riff, wo die Art auch im Vorriff noch häufig ist. Auch Sarcopbyton glaucum ist in erster Linie der hellsten Lichtzone zuzurechnen, doch ist es auch in der anschließenden Zone noch verbreitet, was das Vorkommen im Vorriff bestätigt.

Ihrem Helligkeitsanspruch gemäß ist auch Tubularia larynx in diese Zone einzuordnen. Die Art kam allerdings nicht im eigentlichen Lichtorgelbereid (gleichmäßig laminare Störmung!) vor.

(b) Dämmerlichtzone, hell (wenig Oberlicht, Streulicht, 3-1\% der über der Wasseroberfläche gemessenen Beleuchtungsstärke) mit den Leitformen Dendronephtbya bemprichi und Siphonochalina siphonella. Außer den Leitarten sind für diese 
Zone typisch: Callyspongia crassa(?), Halopteris glutinosa, Dendronepbthya utinomii und Stereonephthya cundabiluensis.

Ubertragen in das natürliche Riff entspricht diese Zone ungefähr dem oberen Vorriff von der Basis des Riffabsturzes an (von 8 bis $12 \mathrm{~m}$ Tiefe abwärts). Wie in den Anmerkungen zu den einzelnen Arten schon mehrfach hingewiesen wurde, finden sich aber auch schon weiter oben im Riff vereinzelt Stellen mit diesen Lichtverhältnissen. In jedem Fall handelt es sich dann um Plätze, die meist überhaupt nicht dem direkten Tageslicht ausgesetzt sind und nur sehr diffuses Streulicht erhalten, bei dem der langwellige Anteil schon weitgehend herausgefiltert ist.

(c) Dämmerlichtzone, dunkel (Streulicht, Reflexlicht, 1-0,4\% der über der Wasseroberfläche gemessenen Beleuchtungsstärke) mit Leitformen Acabaria biserialis und Clatbraria rubrinodis.

Die Abgrenzung dieser Zone ist schwierig. Dendronephthya- und Stereonephthyat-Arten sind auch hier noch verbreitet. $\mathrm{Da}$ jedoch die als Leitformen genannten Gorgonarien in der vorherigen Zone höchstens sehr vereinzelt auftraten, scheint die Unterteilung der Dämmerlichtzone in "hell“ und "dunkel“ gerechtfertigt. Nephtbea albida und Scleronephthya corymbosa haben ihre Hauptverbreitung ebenfalls in diesem Bereich. Acabaria pulchra hat die gleiche Lichtindikator-Qualität wie Acabaria biseralis, doch muß hier wie bei Tubularia larynx angemerkt werden, daß die Art nicht im eigentlichen Lichtorgelbereich (vgl. Abb, 2 und 5) vorkam. Entsprechendes gilt für Laomedea dichotoma.

Nach dem Vorkommen dieser Leitformen im natürlichen Riff entspricht dieser Zone das tiefere Vorriff von ca. 15-20 m Tiefe an abwärts. Die untere Grenze konnte durch Tauchabstiege nicht erfaßt werden.

(d) Dunkelzone (Reflexlicht, 0,4-0,1\% der über der Wasseroberfläche gemessenen Beleuchtungsstärke) mit Leitformen Lithothamnion spec. und Litbophyllum spec.

Die Zone ist mit den Leitformen zwar gut gekennzeichnet, doch zählen diese rotvioletten Kalkalgenlager zu zwei taxonomisch sehr schwierigen Gattungen, denen Arten mit sehr unterschiedlichen ökologischen Ansprüchen, insbesondere hinsichtlich der Helligkeit angehören. Somit nïtzt in diesem Falle die Gattungsangabe allein noch nicht viel zur Charakterisierung. Die tierischen Vertreter dieses Abschnittes: Chama spec., Spondylus gaederopus, Polycarpa mytiligera und Pyura momus besiedeln zwar auch diese Zone, weil sie hier weitgehend unbehindert von Raumkonkurrenten sind. Doch sind sie in bezug auf den Helligkeitsgrad unspezifiziert, so daß sie alleine keinesfalls als Indikatorarten speziell für diese Lichtzone herangezogen werden können.

Im natürlichen Riff entsprechen dieser Zone Höhlen oder ähnlich stark lichtabgeschirmte Räume. In keiner bei Tauchabstiegen erreichten Tiefe (bis unter $40 \mathrm{~m}$ ) war das dem Tageslicht exponierte Vorriff (oder der offene Uferabhang) von einer Biocoenose besiedelt, die ungefähr der Dunkelzone der Pfeiler-Lichtorgel entsprochen hätte.

Mit vorliegender Untersuchung ist im Roten Meer ein erster Versuch gemacht, Indikatorarten für die Beleuchtungsstärke im Korallenriff zu ermitteln. Freilich wird sich der Aussagewert bestimmter Arten erst allmählich nach weiteren Vergleichsuntersuchungen an verschiedenen Plätzen und bei unterschiedlichen ökologischen Bedingun- 
gen genau herauskristallisieren lassen. Auf diese Weise werden dann mögliche, durch die einzelne Untersuchungsanlage bedingte Fehlaussagen, wie hier z. B. an den Hafenpfeilern in gewissem Grade bei Millepora dichotoma, Steinkorallen und Lithophyton arboreum, ausgeglichen werden.

Es ließe sich die Abstufung in verschiedene Helligkeitsgrade sicher noch verfeinern, wenn man - quantitativ und statistisch arbeitend - Mengenkombinationen bestimmter Arten bei verschiedenen Helligkeitsgraden vergleichen würde. Das methodisch einfachere Nebeneinanderstellen spezifischer Leitarten (ohne deren Mengenverhältnis untereinander $\mathrm{zu}$ beachten) führte in vorliegendem Falle zu einer Einteilung in vier Zonen. Der Genauigkeitsgrad erscheint bei der zunächst gestellten Frage, ob und in welchem Ausmaße die Helligkeit die Verteilung der Arten im Korallenriff bedingt, allerdings erst von sekundärer Bedeutung.

Entscheidend ist, daß die dominierende Rolle des Faktors Licht bei der Tiefenzonierung im Korallenriff deutlich wurde: Es wiederholte sich nicht nur die vertikale Verteilung der Arten auf den äußeren Stützpfeilern in waagerechter Richtung von den vorderen zu den hinteren Pfeilerreihen. Weit mehr noch hat sich eine repräsentative Vergesellschaftung von Tier- und Algenarten des Korallenriffs, die natürlicherweise in vertikaler Abstufung vom Riffach bis zum unteren Vorriff (in $40 \mathrm{~m}$ Tiefe und mehr) verteilt ist, einzig dem Lichtgradienten zwischen den Kai-Stützpfeilern folgend in horizontaler Richtung angesiedelt. Außer der Helligkeit hatten dabei Faktoren, die sich ebenfalls mit zunehmender Tiefe ändern, wie Druck, Temperatur, Sauerstoffgehalt, Sedimentfracht und Wasserbewegung, keinen Einfluß.

\section{ZUSAMMENFASSUNG}

1. Die Bedeutung des Faktors Licht für die Verteilung der verschiedenen Arten im Korallenriff wurde an einer Lichtorgel untersucht, als welche die Stützpfeiler der $550 \mathrm{~m}$ langen Pier im Handelshafen von Eilat angesehen werden können. Die Pfeiler sind in acht Reihen hintereinandergeordnet, wobei die äußerste Reihe sonnenlichtexponiert ist, während die Helligkeit bei den hinteren Pfeilern der dunkler Höhlen entspricht.

2. Der Lichtgradient zwischen den Pfeilern wurde in sechs Stufen gegliedert: Stufe A $13-5 \%$, B 5-1,6\%, C 1,6-0,8\%, D 0,8-0,4\%, E 0,4-0,2\%, F 0,2-0,1\% der über der Wasseroberfläche gegebenen Beleuchtungsstärke.

3. Die Verteilung von 28 Tier- und 5 Algenarten auf den Hafenpfeilern und im Riff wurde beschrieben. Thre Eignung als Leitarten wurde überprüf. Auf Grund deren unterschiedlichen Lichtbedarfs ergab sich folgende vierstufige Zonierung der Hafenpfeiler: (a) Hell z o ne (13-3\% der Beleuchtungsstärke über Wasser) mit den Leitformen Tridacna maxima und T. squamosa, Padina pavonia und verschiedenen Steinkorallen; (b) Dä m m e $\mathrm{lich} \mathrm{tz}$ on e, hell (3-1\%) mit den Leitformen Siphonochalina siphonella und Dendronephtbya bemprichi; (c) D ä $\mathrm{m} \mathrm{m}$ e $\mathrm{r} 1 \mathrm{ich} \mathrm{t}$ zone, d u $\mathrm{kel}(1-0,4 \%)$ mit den Leitformen Acabaria biserialis und Clathraria rubrinodis; (d) $\mathrm{D}$ u $\mathrm{n} \mathrm{k}$ e $\mathrm{z}$ on e $(0,4-0,1 \%)$ mit den Leitformen Lithothamnion spec. und Litbopbyllum spec. 
4. Tubularia larynx und Acabaria pulcbra wählten Standorte dicht unter der Wasseroberfläche in unregelmäßig bewegtem Wasser: erstgenannte Art in der "Hellzone“, die zweite in der "Dämmerlichtzone, dunkel“.

5. Die Verteilung der Arten im Lichtgradient der Hafenpfeiler stimmt weitgehend mit der im natürlichen Riff überein. Abweichungen wurden diskutiert.

6. Es wurde deutlich, daß bei denjenigen der untersuchten Arten, bei denen keine gleichmäßige Verbreitung im Riff festzustellen war, die Unterschiede im Vorkommen in erster Linie vom Licht abhängig waren.

Danksagungen. Folgende Herren haben freundlicherweise die Determination einzelner Tiergruppen und Algen übernommen: Dr. M. Grasshoff, Frankfurt (Gorgonarien), Dr. Y. Lipkin, Tel Aviv (Algen), Dr. C. Monniot, Paris (Ascidien), Dr. H. E. Schmidt, Gießen (Hydroiden), Dr. M. Tsurnamal, Jerusalem (Poriferen), und Dr. J. Verseveldt, Zwolle (Alcyonarien). Idh möchte mich hier noch einmal sehr für die gute Zusammenarbeit bedanken. Herrn D. Friedman, Eilat, und den Angehörigen des Marine Biological Laboratory, vor allem dem inzwischen verstorbenen Leiter, Dr. H. Sternitz, danke id. für Hilfe bei der Durchführung der Untersuchungen in Eilat, weiterhin Herrn F. SEEWALD, Tel Aviv, für vielfältige Unterstützung bei den Taucharbeiten. Die Deutsche Forschungsgemeinschaft ermöglichte die Untersuchung durch Bereitstellung eines Forschungsstipendiums.

\section{ZITIERTE LITERATUR}

HezLebust, J. A., 1970. Light - Plants. In: Marine ecology. Ed. by O. Kinne. Wiley-Interscience, London 1 (1), 124-158.

Jerlov, N. G., 1970. Light - General Introduduction. In: Marine ecology. Ed. by O. KinNe. Wiley-Interscience, London 1 (1), 95-102.

Ktausewitz, W., 1967. Die physiographische Zonierung der Saumriffe von Sarso. Meteor Forsch.Ergebn. (D) 2, 44-68.

Kolkwitz, R., 1909. Okologie der tierischen Saprobien. Beiträge zur Lehre von der biologischen Gewässerbeurteilung. Int. Revue ges. Hydrobiol. Hydrogr. 2, 126-152.

LoYA, Y., 1972. Community structure and species diversity of hermatypic corals at Eilat, Red Sea. Mar. Biol, 13, 100-123.

- \& Stobonkin, B., 1971: The coral reefs of Eilat (Gulf of Eilat, Red Sea). Symp. zool. Soc. Lond. 28, 117-139.

MERGNER, H., 1971. Structure, ecology and zonation of Red Sea reefs (in comparison with South Indian and Jamaican reefs). Symp. zool. Soc. Lond. 28, 141-161.

- \& Schunmacher, H., 1973. Untersuchungen über die physiographische Zonierung von Korallenriffen bei Aqaba (Rotes Meer). Helgoländer wiss. Meeresunters. 25 (im Druck).

RuedL, R., 1966. Biologie der Meereshöhlen. Parey, Hamburg, 636 pp.

Yonge, C. M., 1963. The biology of coral reefs. Adv, mar. Biol. 1, 209-260.

Anschrift des Autors; Dr. H. Schuhmacher

Lehrstuhl für Spezielle Zoologie

Ruhr-Universität Bochum

463 Bochum

Postfach 2148

Bundesrepublik Deutschland 